\title{
Construction of the Policy Network Analysis Framework for the Implementation of China's Higher Education Informatization Policy
}

\author{
Yuan Liang*, Lei Zhang, Zijing Wu \\ School of Humanities and Law, Northeastern University, Shenyang 110169, China \\ *Correspondence Author: liangyuan@cse.neu.edu.cn
}

\begin{abstract}
The research on higher education informatization policy is actually a theoretical abstraction of realistic policy implementation, and it is also a research direction that is fully applicable to China's local application and theoretical interpretation. Due to the special nature of education policy, it is not advisable to explain policy results purely based on actors or structural characteristics of the policy network. It should be more in line with the social reality of multi-party's full participation in benefit games, multiple social organizations, and the social difference pattern of China, and step from the causality between a specific policy network and a specific policy result towards a concrete policy network interpretation framework.
\end{abstract}

Keywords: Higher educational informatization policy, Policy network, Analysis framework.

\section{Introduction}

As policy complexity and uncertainty continue to increase, the policy network has gradually become the main paradigm for policy analysis by policy scholars. After more than forty years of extensive and in-depth research, the policy network perspective can explain the dynamicity and complexity of policy implementation well. However, there are still many theoretical divergences regarding the policy network analysis framework, especially whether it can explain and predict policy results or not, which is one of the important foundations for the framework to become a theory. Policy network theory is both an explanatory and constructive framework, which can construct a policy process with practically operational significance. This article will do a general analysis of the existing policy network analysis framework and method, proposes the "structure-action-results" revision model of the implementation of higher education informatization policy.

\section{The Existing Policy Network Analysis Framework Review}

Through the debates of policy scholars and the continuous revision of the policy network analysis framework, the "structure-result" analysis logic represented by R.A.W. Rhodes and Marsh, the "behavior-result" analysis logic represented by Dowding and Macpherson and the network management framework represented by Klijn have gradually formed.

\subsection{The "Structure-result" Analysis Logic}

The "structure-result" analysis logic represented by Rhodes gives structure privileges and is full of structuralism, trying to take a neutral path. He believes that governance refers to the inter-organizational network of self-organizations and consider governance as "minimizing the state", "social-cybernetic system" and "self-organizing network". Governance stresses the interdependence between organizations, the continuous interaction between network members, game characteristics, a high degree of autonomy not controlled by the state and the influence of the network structure on policy outcomes. Rhodes accepted Benson's point of view and proposed a policy network analysis framework based on the theory of power dependence, focusing on the analysis and discussion of the policy formulation process. He points out that the analysis of the policy network is based on the interdependence of network actors' resources. Any organization depends on another organization to provide resources that it lacks and organizations must exchange resources with one another in order to achieve their own goals. The key to the discretion of an organization lies in the relative power potential between organizations and their respective goals. He also brings up the concept of "advanced alliances" that has a larger space to explain policy issues and has the ability to adopt various tactics to regulate the process of resource exchange under the existing "rules of the game".

In terms of changes in the policy network, Rhodes and Marsh suggest that the degree of policy changes depends on the significance of related issues and that the changes are gradual. In the network affected by frequent interaction of network members, the goals pursued by the network, the tools to achieve the goals and the network standards will gradually become institutionalized and counter-control the behavior of network entities. He also indicated that in closely-organized and relatively-closed networks, for example, policy community networks have frequent influences while issue networks are relatively unconsolidated and have a relatively low degree of institutionalization, network interaction has less impact on changes.

Marsh and Smith amended the limitations of the policy network's lateral emphasis on the structure, trying to resolve the relationship between macro and micro variables through dialectical approaches, mainly emphasizing three pairs of dialectical relationship: "network structure and actors", "network structure and environment", "policy network and policy outcome". The dialectical relationship between network structure and actors mainly stresses that structure is 
still the main indicator of network relationship. Structure affects the network structure and the use of actors' resources, reflects the environment, actors' resources, network interaction and policy results, and defines network actors, issue preferences, resolutions, organization rules etc. However, the policy network structure and resource dependence of the policy network are not invariable. Individuals can influence the action strategies of other network members via their dynamic roles to adjust the structure of the policy network which reflects the interaction of actors' resources, skills, network structure and policy, and actors' skills are also derived from innate cognition and acquired learning. The dialectical relationship between network structure and environment mainly underlines that the exogenous variables of the policy network changes come from the environment, including politics, economy, culture, ideology, etc., especially the restrictions and changes of the political environment, which will prompt network actors to change their personal cognition structure resulting in changes in social psychology and values, and in turn forming a new structure and norms of interpersonal relationships. These are changes of the internal network resulted from the external environment. The dialectical relationship between the policy network and policy results mainly lays stress on the reciprocal influence between the policy network and policy results. Policy results reflect the interaction between the network structure and the policy network itself and policy makers will be affected by the policy network, producing relevant policy outcomes on the basis of common policy preferences. On the other hand, the policy results also have a counter-effect on the policy network-network actors will update their action methods and strategies because they have not obtained the benefits from the policy results, and the policy results will also destroy the balance of internal benefits and resources of the network actors.

\subsection{The "Behavior-result" Analysis Logic}

The "behavior-result" analysis logic represented by Duding, based on rational choice theory, criticized the ambiguity of the traditional policy network analysis in the concept and role of variables. The article "Model or Metaphor? Criticism of the Policy Network Path", published by Dowding, thoroughly criticizes and denies the policy network analysis path, pointing out that the policy network analysis path might be a useful "metaphor" rather than a rigorous analysis model, that is, at a certain point in the policy process, the network relationship "described" by the policy network path often becomes a metaphor of "naive and unconscious interactive view" pulling network actors from social structure, which triggered the big debate between Marsh and him. Dowding argues that the influence of the policy network on the policy results should focus more on the main role of the network actors during the policy process and the actors should be used for analysis instead of taking policy network structure and characteristics itself as the independent variables. He pointed out that the policy outcome is determined by the bargaining of network members rather than a certain policy network. The driving force of policy outcome changes comes from the policy network components, which explain the nature of the policy network and the policy process simultaneously. The interactive behavior between network actors cannot be ignored for the reason that it affects policy results. The descriptive network structure itself will not affect policy results. The changes in policy results must be illustrated according to the internal changes in the resource dependence mode in the network. Even McPherson and Raber also agree that policy results also depend on interpersonal interaction, which makes resource exchange possible, determining the degree of influence of network actors in the policy process as a result. They use the analytical concept of individualism, and the actors themselves have personal networks of actions and the possibility of interpreting the structure. Raber suggests that the analysis of the policy network should be directed towards understanding the characteristics of the actors, especially how, when, where, why to enter the policy network, and what bargaining methods should be adopted to understand the policy network. Individual strategies construct the structure of the network and the structure affects the policy outcome in turn. But Dowding's analysis focuses on the actor's purposeful behavior, puts the actor's intention and initiative in the first place and assumes his preferences, ignoring the influence of the network structure relationship, and that the individual's interpretation of the network structure is also affected by the network structure itself, which reflects the duality of structuralism and behaviorism.

\subsection{Network Management Analysis Framework}

From a macro analytic perspective and based on the research of policy network management strategies, the network management analysis framework represented by Klijn proposes to rebuild the policy network to optimize the network and ultimately reach the goal of improving the efficiency of policy network operation and achieving the result of the ideal governance effect. The three elements of network management are the intervention of the existing relationship model, or rather, network relationship reconstruction, consensus building and problem solving. After repeated research and modification, Klijn finally used the four dimensions of actors, cognition, resources, and systems to build a network management analysis framework which includes two different strategies: game management strategy and network construction strategy. Besides, he put forward a clearer strategy based on empirical research. The game management strategy lays stress on creating the actors' consensus of problems and solutions with the management strategy of the governing subjects when rules, actors, resources, standards and values are already established, while the network construction strategy focuses on establishing or changing the institutional arrangements that the policy network consists of to make it easier for actors to cooperate. Afterwards, Klijn also made some amendments to the network management framework, including merging resource and cognitive dimensions into the same dimension of actors, adding the analysis dimension of the relationship between actors based on the network structure, and increasing the dimension of network managers. The adjusted policy network management analysis framework is relatively simplified one, including four dimensions: network manager analysis, actor management, network structure-based relationship management between actors, and network rules management. The academic community holds the view that the addition of the analysis framework of the network 
structure can make up for the lack of research on the relationship between actors in the previous two generations of policy network, strengthen the research on the significance of the relationship between network actors, and even guide the value and cognition of the target group and influence the value, rules and cognition through "internalization". Scholar Carlsson regards the policy network as collective action, demonstrating the similarities between the structure of the policy network and the four types of collective action; Smelser establishes the connection between collective action and the policy network through the supporting analysis of value accumulation theory.

\section{The Analytical Method of Policy Network Theory}

The analytical methods of policy network theory also vary according to the analytical framework constructed by scholars, mainly highlighting the influence of network interaction on the policy process rather than explaining the causal relationship between network types and policy results.

Rhodes's traditional institutional method, or the classic institutional method, is the description-inductive method, the formal-legal method and the historical-comparative method based on the institutional analysis approach. Currently the three types of system analysis are: constitutional research, public administration, and "new institutionalism". The key feature of constitutional research is that it is still the birthplace of formal-legal approaches and liberal-democratic reformism. Johnson contends that institutional analysis does not adopt a limited view. What it analyzes is actually the system in action, and it treats the system as an expression of political goals. Dearlove reckons that constitutional research should be regarded as "an explanatory starting point" under a more extensive theoretical framework of national research. Rhodes believes that in order to avoid formalism, constitutional research must be put into a broader perspective or theory. The key feature of public administration is system research, and Robson thinks that it focuses on local public administration institutions, analyzing their history, functions, powers, and relationships, and exploring how these institutions operate and the degree of benefits they have achieved. Organizational theory is a solid constituent of the history of public administration knowledge, the key characteristic of which is to focus on formal organizations all the time. In "New Institutionalism", Hall aims for creating a historical-institutional interpretation that "can explain the historical continuity of policies and transnational changes". He clarifies that "new" institutionalism is the encounter between history and organizational theory for the purpose of studying political systems. The policy network is a "state-centered" research approach to public policy formulation and studies "behavior in an institutional environment". Therefore, policies originate from the interaction between government organizations (especially central government departments and institutions) and a network comprising other organizations (especially professional interest groups and economic interest groups). The policy network is an approach to research on institutionalism.

Borze puts forward two different analysis paradigms: quantitative and qualitative network analysis, as well as the policy network type of interest intermediary and special governance. The quantitative and qualitative network approach uses the network as an analytical tool. The quantitative approach takes network analysis as the method of social structure analysis, and use quantitative methods, such as hierarchical cluster analysis, density tables, and cut-off models. It analyzes the relationship between participants according to the coagulability of participants, the equivalence of structure and the representation of space. The qualitative approach not only pays attention to the structure of participant interaction, but also pays more attention to the trend of the process. It uses qualitative methods, such as in-depth interviews, content analysis, and speech analysis, to understand the interactive network. The comprehensive utilization of qualitative and quantitative analysis can make up for each other's deficiencies and obtain more accurate analysis results and utility. Relying on the distinction between the interest school and the governance school of policy network theory, it can be divided into two types of analysis: the interest intermediary type and a special form of governance network. The interest intermediary school believes that the policy network is a collective term for the relationship between various interest groups and the state; the governance school believes that the policy network is a specific form of governance and a political resource mobilization mechanism. Interest intermediary is a concept focusing on quantitative measurement, while governance is a more quantitative concept of policy network. According to Borze, the policy network of interest intermediary and governance can be an analytical tool and a theoretical approach. However, the interest intermediary school mostly examines sectoral policy-making and sub-sectoral policy-making from the perspective of analytical tools. According to Borze's classification, it can be summarized as a quantitative policy network concept intermediary method and a qualitative policy network concept governance method.

Bob Jessop brings up a "strategy-relationship" analysis method. Subjected to the standards and restrictions of both the specific historical location of Time and Space and the spatial organization of politics and economics, he views capitalist society as an ecological system and reckons that the country's strategic choice is a combination of both the system structure and the struggle of the country's internal and external social forces. The relationship between "structure" and "activity" is well figured out with the help of the theoretical framework of "structure-activity". In this way, the country becomes an unbalanced arena, which sometimes favors certain actors, but may become more beneficial to other actors over time. Jessop replaced the concepts of "structure" and "activity" with "strategic choice context" and "strategic behavior", surpassing the possibility of the dualism of "structure" and "activity". The principal relationship of this analytical approach is the more direct interaction between strategy actors and their strategic environment and context.

Besides, Chinese scholars also put forward their own insights on policy network analysis methods. Guangxu Wang proposed a policy network analysis that returns to the nature of the network. As an interpretation method of middle-level theory, network analysis provides a moderating thinking 
direction for the dual opposition between macro and onlookers in social sciences. Network analysis abandons the dualistic thinking, and is unique in that it has the characteristics of "anti-classification laws"-it opposes any interpretation of human behavior or social processes based solely on the attributes and behavior of actors. What the network analysis needs to answer is: how and how much influence the behavior of network members are affected by the pattern of relationships among various actors. The network analysis observes individuals or organizations placed in the network structure, stressing the importance of the relationship between people or between organizations and organizations. The network analysis method is intended to describe an image of the interaction mode of actors, using concepts, such as frequency, degree of centralization, and density, to illustrate their relationships. The core concept of the policy network is the interdependence between actors in order to influence policy output. Liping Sun uses the "process-event" analysis to analyze the relationship between the state and peasants in contemporary China or the relationship between the state and society in the countryside. It is an improvement on the traditional "structure-system" analysis method, breaking through the limitations of static structural analysis and helping to enhance the understanding of the traditional social structure. It offers a better solution to the problem of the interaction and relationship between the state and society in the rural areas of our country and explores the practical form of the relationship between the state and peasants. Based on the complex and subtle relationship between different things or different factors within the same thing, "Process-Event Analysis" is a dynamic process in which the researched object is communicated by a static structure, special items, to a dynamic process communicated by several events. And can only be fully demonstrated through events or processes. This analysis method provides a dynamic perspective to look into the relationship between the state and society with an understanding of the subtle mechanism, and reveals the complex relationship between towns, villages, and farmers through the analysis of specific event processes. Jing Zhang adopted "Structure-Institutional Analysis" and was influenced by the "New Institutionalism" school to respond to Liping Sun 's criticism of "Static Structural Analysis". "Structure-Institutional Analysis" thinks highly of the social rules of behavior, especially the social structure or social relationship reflected by specific "events" or "processes". Different systems will stimulate different behaviors and different events. The substance of events occurrence and system changes stems from the power relations and structural changes of different groups of people. She also made it clear that the "system" in the mentioned analysis method includes the two concepts of "true system" and "non-true system", and suggested trying to choose different analysis methods by "different problems concerned and the nature of the information obtained" to. Lizhong Xie combined the analytical perspectives of the two scholars mentioned above and put forward the perspective of "multiple discourse analysis", trying to explain the most basic theoretical analysis in the field of sociology, that is, the diverged opinions on the relationship between "society (structure-institution)" and "individual". "Multiple discourse analysis" believes that there is no purely natural "objective reality" that separates from the existence of people's discourse. It is a linguistic existence which is produced by speakers under the guidance and limitation of some certain linguistic system and abiding by specific rules of discourse formation. Speakers under the constraints and guidance of different discourse systems may have different constructions of "reality".

\section{The Policy Network Interpretation Framework for Higher Education Informatization Policy Implementation}

\subsection{The Establishment of a Revised Model Based on the "Structure-Action-Rresult" View}

In "Xunzi The System of Kings", it is said that the principle of organizing social groups is appropriate, then everything can be properly placed, that is to say, organizing people into a collective or a society is where the fundamental function and essential regulation of the leader lies. Xiaotong Fei also pointed out that a society of differential order is a network of countless personal relationships, each node of which is attached to a moral element. China's morals and laws are all adjusted to the extent correspondingly in light of the relationship between the target and the "self". There are two views on power: One is from the perspective of social conflict, power is manifested in the form of master and slave among different groups or classes in society. The other one is originated from the perspective of social cooperation, the foundation of power is the social contract, or rather, consent, which is called "consent power."

In the context of education democratization in China, to achieve the common goal of higher education informatization, further theoretical construction, value analysis, tool updates, and case studies will be carried out combined with social network analysis methods. While exploring the significance of relationship, higher education informatization will be considered as an educational governance action with dual attributes of government behaviors and social activities and the requirements of various interest groups in subject education will be fully satisfied. Moreover, actors' strategies will be highlighted and policy deviations will be corrected in a timely manner.

Therefore, under such a modified perspective, this research combines the "structure-result" analysis logic, "behavior-result" analysis logic and network management analysis framework of the previous research results, and proposes the "structure-action-results" revision model of the implementation of higher education informatization policy. In other words, it is an educational policy execution network analysis framework composed of a static structure layer, a dynamic action layer, and a result layer. "Structure" refers to "static structure layer", including the policy network environment (nature, society, economy, information technology, institutional background, etc.) of the implementation of higher education informatization policy and the network structure and interpersonal relationship structure of the policy network itself, namely, the relationship between education policy actors. "Action" is short for "dynamic action layer", including the strategy skills and network interaction of multiple actors in the 
implementation of higher education informatization policy. "Result" means "result layer", which refers to the impact of higher education informatization policy implementation on the policy process, policy results, and the overall education governance model.

\subsection{Analysis of Analytic Framework}

4.2.1 Static structure layer: the macro network environment and network structure of policies

The static structure layer describes, interprets, and analyzes the policy network environment for the implementation of higher education informatization policy, the meso-level network structure, and the unique interpersonal relationship structure of Chinese society in a macro aspect. Derived from the analysis of the relationship and location between actors, it uses social network analysis approach to analyse the relationship between actors in a policy network, trying to find out the strategies that individual behaviors actually present under the structure of the policy network.

In light of its close connection with information technology, higher education informatization policy will inevitably focus more on the dialectical interaction with the environment. The natural environment, social environment, economic environment, information technology, institutional background and other policy network environments have become exogenous variables causing the formation and changes of the policy network, by exerting influence on the supply and utilization of network actors' resources and leading to changes in the network structure. Meanwhile, China's social transformation is accelerating, the interest structure is diversified, and the policy process is changing rapidly. The informal policy relationship network in the process of higher education informatization policy implementation also has some "personalized structure" influence and deviation resulted from "relationship dominance" on the effect of policy implementation.

The policy network structure is the internal or external relationship model between multiple and heterogeneous network actors. According to the policy network types summarized by the Roots model, it is divided into the policy community, the intergovernmental network, the professional network, the producer network and the issue network, all of which constitutes a diverse network structure. Elements like the difference of network types, the degree of connection between nodes will affect the ultimate policy outcome of higher education informatization, and the resources and rights occupied by each network and the corresponding responsibilities are not equal, both complementing and restricting each other. The actors in the higher education informatization policy network are more directed towards educational governance entities, such as the government, universities, social organizations, experts, parents, media, etc., forming a policy community centered on the Ministry of Education and the government, an intergovernmental network centered on universities and local education authorities, a professional network centered on expert think tanks, and a topic network centered on college teachers and students, parents, media, and social organizations.
4.2.2 Dynamic action layer: the micro-actor of policy and network interaction

This layer is searching for the technique of network interaction action and strategy based on the perspective of the micro-actors of policy. Actors of higher education informatization network possess diversified resources and powers. With the development of technology of information and communication, interpersonal networks are enriched and attach humans with other networks. All the behaviors that people rely on network leave marks, and the network makes " $1+1>2$ " possible. Actors' connectivity nature, known as the structural features of the network, including features like the density, magnitude, frequency of the network and traits like the occupants of the overall structural hole's best position, will affect policy agenda, innovation and transition.

However, the cooperation among networks makes up the education policy network mother-system with existed communication approaches. Multi-actors will utilize their resources and schemes to affect the policy community that makes the ultimate decision, use the network structure to aggregate advantageous resources and exchange information and realize the policy target eventually. There are both internal and external network interaction in the implementation of higher education informatization policy. Internal network interaction is the interactive process of different educational policy actors based on resource interdependence while the external counterpart refers to the interaction of different network structures during the implementation of higher education informatization policy. The implementation of high educational informatization policy is conducted with the interaction of policy actors, who form compact interactive network by establishing locations in the network, formulating action rules, and ascertaining policy issues and solutions through interdependence. The implementation mode of high educational informatization policy is a process of realising good governance through interactive balance, emphasizing the participation and interaction of multi-actors and combining "structure interaction" and "governance interaction".

Higher education informatization policy network also requires returning to think over the nature of the network itself and analyzing according to the social network. It stresses the ability of actors, namely nodes, to act autonomously for the perceptual target interests under the control of the network context. The interactive relation of each network has its own particularity. The flow of resources between nodes, the direction of flow, and the frequency of resources exchange must be determined in line with the interactive relation between the purpose of the network and the actors. "From the perspective of the Internet, the power conferred by the formal system will be penetrated in the process of network interaction. Power is clarified through the structure of interdependence of resources in the policy network, which is different from the adherents of institutional theory in a sense."

\subsubsection{Result layer: effects and feedbacks of the policy}

The result layer concentrates on the effect of policy resulted from the interaction between the actors and the policy 
network of higher education informatization policy. Effects of the policy will have a feedback on the network structure and the actors, even affecting making policies. In such a practical path of higher education informatization policy implementation, the result layer lays stress on the transition and circulation of education policy information. Actors study policies with the strategic information acquired from specific structure and effects of the policy. Multi-actors' ability of policy learning will enhance their flexible utilization of policy tools and facilitate the access of internet resources, influencing policy making and implementation further. Effect of the policy and the actors learning process are continuous and reciprocal, when actions have an impact on the actors' strategies and effects of the policy, the results will influence personal action in the future in return.

The effect of policy will make policy makers attach more importance to policy network learning and take full consideration of the fact that the network pattern of higher education informatization policy implementation is a democratic one under the scope of the policy network. Fully discovering the respective interest requirements behind every actor's behavior, narrowing the distance of various interest entities' requirements, locating the problem and providing corresponding solutions, help avoid the deviation in policy implementation. According to Zhu Chunkui, there are at least three aspects where effects of the policy may affect the policy network: specific effects of the policy might result in the change of network members and the balance of internal network resources; effects of the policy might influence social network structure and diminish special interest of existing networks; effects of the policy will influence personal actions.

From the perspective of governance, the policy network is considered as a way of governance which is often be applied to adjust the interactive relationships and network structure among actors to the advantage of the output of effects of the policy.

\section{Conclusion}

Apparently, it is decision that takes the central part in China's society, rather than the multi-variants decision structure in some countries. By constructuring the analysis framework of policy network theories, both the process of how policy implementation affects policy results and the interaction and influence of public interest departments and special interest groups during policy process can be well illustrated and interpreted. What's more, as a relation-type society, the complexity of policy relationship network brought about by personification structure is higher than counterparts. Therefore, while formulating the explanatory framework of higher education informatization policy, the big background that China is experiencing profound social changes and economic transition should be taken into account as well as the pattern of difference and Chinese relation-type social reality.

\section{References}

[1] R.A.W. Rhodes. Understanding Governance: Policy Networks, Governance, Reflexivity and Accountability
[M]. Beijing: China Renmin University Press, 2020, 6: 56-59, 70-73.

[2] Klijn E H. Analyzing and Managing Policy Processes in Complex Networks: A Theoretical Examination of the Concept Policy Network and Its Problems [J]. Administration \& Society, 1996, 28(1): 32.

[3] Yapeng Zhu. An Analysis of Policy Network: Development and Theory Building [J]. Journal of Sun Yat-Sen University (Social Science Edition), 2008(48): 192-199.

[4] Marsh D, Smith M. Understanding Policy Networks: Towards a Dialectical Approach [J]. Political Studies, 2000(1): 4-21.

[5] Guangxu Wang. The Core Position an Methodological Mismatch of Policy Network Research in the Field of Public Administration [J]. Journal of Political Studies, 2005(5): 61-102.

[6] Dowding, Keith. Model or Metaphor? A Critical Review of the Policy Network Approach [J]. Political Studies, 1995 (XLIII): 136-158.

[7] Liping Sun. Process-Event Analysis and Insights into Contemporary Rural Social Life in Chin [C]. Rural Grassroots Political Power Operation and Villagers Autonomy, Beijing: China Social Sciences Press, 2001: 11.

[8] Jing Zhang. Basic Unit of Political Power: Problems with the Rural System [M]. Hangzhou, Zhejiang People's Publishing House, 2000: 10-11.

[9] Carlsson Lars. Policy Networks as Collective Action [J]. Policy Studies Jouranl, 2000, 28(3): 502-520.

[10] Marsh David. \& Smith Martin. Understanding Policy Networks: Towards A Dialectical Approach[J]. Political Studies, 2000(48): 4-21. 